\title{
Propostas dietoterápicas para crianças com leucemia linfocítica aguda, sob tratamento quimioterápico no hospital de apoio de brasília ${ }^{1}$
}

\author{
Tatiana Serpa Guedes² \\ Jaime Rodrigues Júnior ${ }^{3}$ \\ Bruna Toscano 4
}

\section{Resumo}

O objetivo deste estudo é propor intervenções nutricionais que possam favorecer a aceitação de dietas hospitalares por crianças em tratamento de Leucemia Linfocítica Aguda (LLA), no Hospital de Apoio de Brasília (HAB), de modo a beneficiar o estado de saúde, o prognóstico e a qualidade de vida desses pacientes. Nesta pesquisa, foi estudada uma amostra composta por 20 crianças, com idade entre 1 e 10 anos, sob tratamento quimioterápico na Ala de Hematologia Infantil do HAB. Foi aplicado aos responsáveis pelas crianças um questionário para verificar as preferências e hábitos alimentares dos pacientes quando internados. Os resultados deste trabalho mostraram que os alimentos mais consumidos pelas crianças, durante o período de internação, foram os lanches rápidos, entre eles frutas, iogurte e pão francês, e os menos consumidos, os alimentos como carnes, verduras, arroz e feijão, que constituem as principais refeições do dia, almoço e jantar. Esses dados sugerem uma aceitação da dieta hospitalar baixa e insuficiente para atender a demanda energética e nutricional dessas crianças, o que pode comprometer, ainda mais, seu estado de saúde e prognóstico.

Palavras-chave: Leucemia. Terapia nutricional. Crianças. Nutrição.

\footnotetext{
${ }^{1}$ Esta pesquisa foi realizada no Programa de Iniciação Científica do UniCEUB.

${ }^{2}$ Bolsista de Iniciação Científica do CNPq - Centro Universitário de Brasília - UniCEUB, Brasília, DF.

${ }^{3}$ Professor Livre-Docente da Disciplina de Patologia, FACES - Centro Universitário de Brasília UniCEUB, Brasília, DF.

${ }^{4}$ Professora Livre-Docente da Disciplina de Dietoterapia, FACES - Centro Universitário de Brasília UniCEUB, Brasília, DF.
} 


\section{Introdução}

A Leucemia Linfocítica Aguda é o tipo de câncer mais freqüente na infância, com uma incidência máxima em crianças entre três e cinco anos. $\mathrm{Na}$ ausência de intervenção médica e nutricional adequada, essa doença pode provocar a morte dentro de poucos meses. Sua sintomatologia caracterizase por fraqueza, anemia, febre, feridas na boca, dor óssea, dor articular e sangramento, podendo, em alguns pacientes, evoluir paracomplicações mais sérias (BARACAT et al., 2000).

Além do impacto fisiológico e psicológico da doença no paciente, ainda existem os efeitos colaterais da própria quimioterapia, que consistem de anorexia, náuseas, vômitos, estomatite, boca seca, alteração do paladar, perda de peso e alterações gastrintestinais como diarréia e constipação (IKEMORI et al., 2003; SHILS et al., 2003). Associados a um conjunto sintomatológico da própria doença, esses efeitos causam desconfortos que se tornam, muitas vezes, um obstáculo à alimentação adequada do paciente. Por essa razão, este pode vir a desenvolver anorexia, aversões alimentares diversas e/ou sensação de saciedade precoce, que contribuem, significativamente, para o decréscimo de sua ingestão alimentar (BRASIL et al., 2003; WAITZBEG, 2004).

Uma menor aceitação da dieta hospitalar pode comprometer o estado nutricional da criança, prejudicar a resposta ao tratamento oncológico, aumentar a toxicidade, os efeitos colaterais e desconfortos causados pela quimioterapia, bem como as complicações, o tempo de permanência hospitalar e os custos. Além disso, e principalmente, a qualidade de vida do paciente e seu prognóstico (WAITZBERG, 2004) podem ser afetados.

Assim sendo, com base nos dados recentes sobre as dificuldades que acercam a ingestão de alimentos e a manutenção do estado nutricional nesses pacientes, este trabalho objetiva desenvolver propostas de intervenção nutricional que possam favorecer a aceitação alimentar e, ao mesmo tempo, suprir as necessidades nutricionais de crianças com LLA, durante o período de internação para quimioterapia, para melhorar sua qualidade de vida e prognóstico. 


\section{Metodologia}

Participaram do estudo pacientes com idade entre um e dez anos, de ambos os sexos, sob tratamento de LLA no Hospital de Apoio do DF. Durante o período entre os meses de dezembro de 2006 e fevereiro de 2007, foram aplicados aos responsáveis de 20 crianças que estavam ou já estiveram em tratamento quimioterápico no Hospital de Apoio do DF, um questionário objetivo e qualitativo, incluindo 16 perguntas relacionadas às preferências e hábitos alimentares dos pacientes durante a quimioterapia (Apêndice A). Esse questionário foi aplicado sob a forma de entrevista, com duração média de 10 a 15 minutos. Para a tabulação dos dados obtidos, foi utilizado o programa do Microsoft Excel 2003.

Foi elaborado, também, um relatório contendo as principais observações registradas durante a pesquisa de campo, relevantes a este trabalho. $\mathrm{O}$ relatório consiste de informações coletadas, diretamente, com duas nutricionistas responsáveis pela Ala de Hematologia Infantil do Hospital de Apoio de Brasília e, ainda, da observação da rotina alimentar e do cardápio oferecido às crianças sob quimioterapia no mesmo estabelecimento. Os dados obtidos com as nutricionistas foram coletados por meio de um questionário constituído por perguntas subjetivas, referentes ao impacto nutricional da quimioterapia em crianças com LLA (Apêndice B) e às principais condutas dietoterápicas adotadas no hospital (Anexo 1).

\section{Resultados}

Os resultados da análise e interpretação dos dados coletados neste trabalho mostram que o nível de aceitação das dietas hospitalares foi baixo, sendo os alimentos mais consumidos pelas crianças, durante o período de internação, os lanches rápidos (Figura 1), entre eles frutas, iogurte e pão francês. Os menos consumidos foram as carnes, as verduras, o arroz e o feijão, principais componentes do almoço e do jantar (Figura 2). 


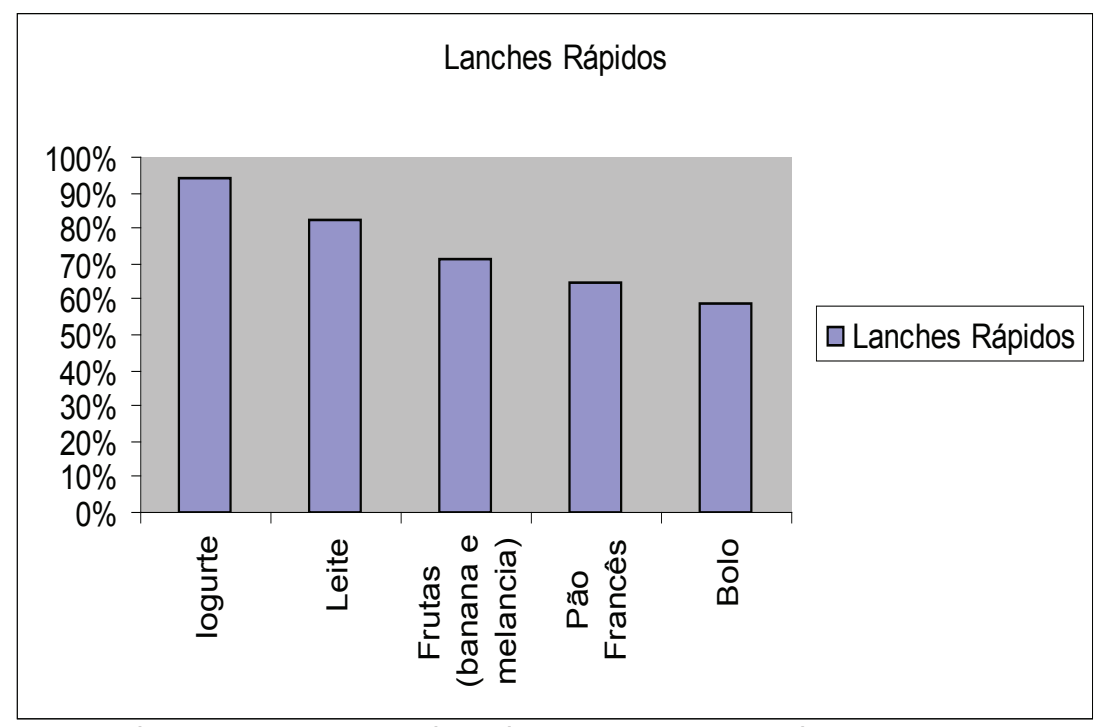

Figura 1 - Alimentos mais consumidos pelas crianças com LLA, sob quimioterapia no HAB

Os dados mostram que, nas principais refeições, almoço e jantar, houve um índice de rejeição de 65\%, ou seja, um valor significativamente alto. Tal fato corrobora com a constatação das nutricionistas do HAB e com dados da literatura que apontam para a dificuldade que acerca a ingestão alimentar de pacientes com câncer, sob quimioterapia (WAITZBERG, 2004).

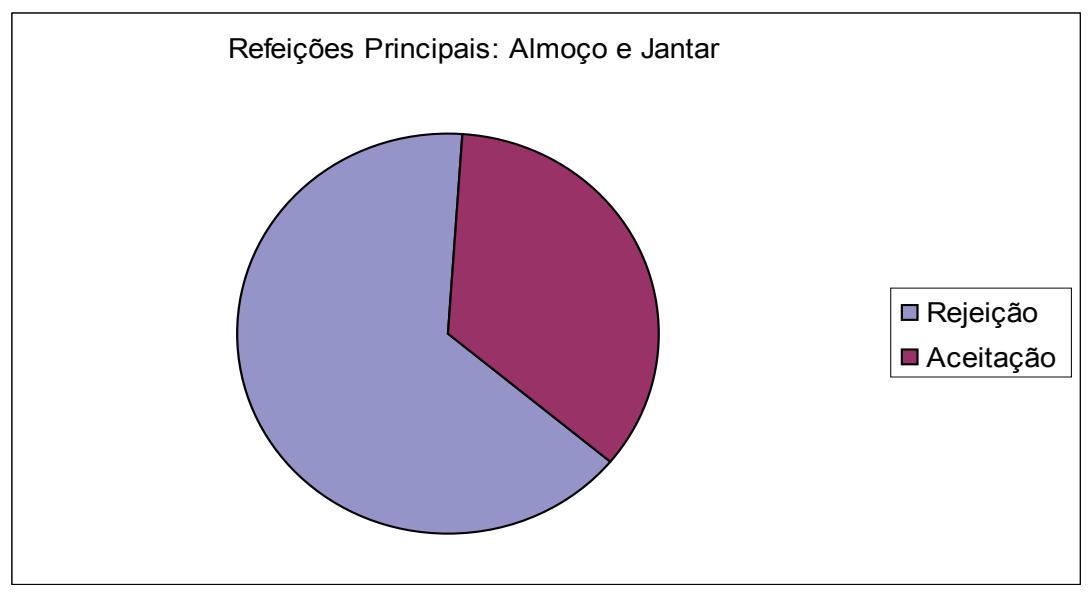

Figura 2 - Índice de aceitação das principais refeições pelas crianças com LLA, sob quimioterapia no $\mathrm{HAB}$ 
Segundo depoimentos dos responsáveis pelas crianças e das nutricionistas, uma das causas de rejeição das principais refeições do dia é decorrente dos próprios efeitos colaterais da quimioterapia, principalmente as náuseas e vômitos que começam, muitas vezes, antes mesmo da refeição ser iniciada. Pôde-se observar que as crianças, freqüentemente, reportam aos responsáveis e às nutricionistas que o odor forte, característico do almoço e do jantar, é, por si só, nauseante. Como conseqüências, as crianças, freqüentemente, desenvolvem aversão a estas refeições, pois temem sentir-se desconfortáveis após o seu consumo.

Essas observações corroboram as declarações de Stump (1999), Baracat (2000), Cuppari (2005) e Mahan (2005), quanto aos efeitos colaterais do tratamento quimioterápico consistir em um dos principais obstáculos à alimentação adequada do paciente. A típica rejeição do almoço e do jantar pela maioria das crianças com LLA, sob quimioterapia, também condiz com as observações de Waitzberg (2004) sobre o efeito das aversões alimentares adquiridas por esses pacientes, em relação ao decréscimo da ingestão de alimentos.

Apesar de serem as náuseas e vômitos duas das principais entre as possíveis causas da baixa ingestão alimentar dessas crianças, dados da literatura (IKEMORI et al, 2003; PINHO et al, 2004; WAITZBERG, 2004) e observações de campo sugerem que as causas são multifatoriais. Uma delas pode estar relacionada à própria contextualização do alimento. As refeições das crianças internadas com LLA são realizadas na própria sala de quimioterapia ou no próprio leito, ou seja, no mesmo ambiente onde elas passam pelo tratamento. Segundo Accioly (2004) e Waitzberg (2004), uma contextualização negativa como a de hospitalização pode ser associada ao alimento ou ao momento da refeição, contribuindo para sua rejeição.

Uma outra possível causa pode ser referente à apresentação das refeições, que são pouco atrativas às crianças. Dados da literatura (MC GRATH, 2002) sugerem que a maneira como as dietas hospitalares são apresentadas aos pacientes pode ser um agravante na aceitação dos alimentos por eles. Accioly (2004) acrescenta, ainda, que dietas que estimulem o aspecto sensorial do alimento podem favorecer maior aprovação e aceitação das dietas hospitalares pelas crianças.

É possível, também, que os sucos oferecidos junto às refeições contribuam para a menor ingestão alimentar dessas crianças. De acordo com Baracat (2000) 
e Mahan (2005), a ingestão de líquidos durante as refeições pode contribuir com para anorexia, náuseas e vômitos, por aumentar a sensação de plenitude gástrica e saciedade.

Por outro lado, os resultados apontam para uma aceitação maior de lanches rápidos, compostos por pão, iogurte, bolo, leite e algumas frutas. Segundo o relato de responsáveis e de nutricionistas, esses alimentos parecem causar menor desconforto gastrintestinal nas crianças, além de serem menos nauseantes. No entanto, esses alimentos, por si só, não podem suprir as necessidades nutricionais e energéticas das crianças, uma vez que contêm pouca variedade nutricional e baixa densidade calórica (PINHO, 2004).

Os pacientes com LLA, com idade entre um e dez anos, encontram-se já, imunológica e fisiologicamente, debilitados, em decorrência da própria patologia (ROBBINS, 2000) e do tratamento com as drogas antineoplásicas, que atingem também as células normais (WAITZBERG, 2004). Além disso, estão em uma fase de crescimento e de desenvolvimento, na qual, as demandas energéticas e nutricionais encontram-se elevadas (ACCIOLY, 2004). Portanto, a baixa aceitação de alimentos importantes para uma nutrição adequada, como aqueles presentes no almoço e no jantar, pode contribuir para o comprometimento do estado nutricional dessas crianças, prejudicando sua resposta ao tratamento oncológico e seu prognóstico.

Assim sendo, os resultados apontam para a necessidade emergente de intervenções nutricionais que visem a favorecer a aceitação de refeições hospitalares mais nutritivas e a melhorar a ingestão alimentar dessas crianças, objetivando suprir melhor suas necessidades nutricionais bem como aumentar a eficácia do tratamento oncológico no combate à doença.

\section{Propostas de intervenção nutricional}

- Melhorar as características nutricionais dos lanches rápidos, acrescentando, nas preparações, alimentos com maior teor de nutrientes, maior densidade calórica e protéica;

- Aliar as prescrições dietéticas e as restrições alimentares dos pacientes com LLA às tendências inovadoras da gastronomia, 
melhorando a apresentação das refeições e tornando-as mais atrativas e saborosas;

- $\quad$ Alterar a contextualização do alimento, substituindo as enfermarias e salas de quimioterapia por novos ambientes, próprios para a realização das refeições;

- Minimizar o odor das refeições do almoço e do jantar, empregando novas receitas que utilizem os mesmos ingredientes em preparações diferentes;

- $\quad$ Retirar os líquidos das principais refeições, repassando-os para os lanches realizados duas a três horas depois.

\section{Conclusão}

No presente estudo, observou-se que crianças com LLA, sob tratamento quimioterápico no $\mathrm{HAB}$, costumam ter maior preferência por lanches rápidos, em detrimento dos alimentos servidos no almoço e jantar. Entre os fatores que mais parecem influenciar essa menor aceitação alimentar das principais refeições do dia estão os efeitos colaterais da própria quimioterapia, principalmente as náuseas e vômitos, que podem tornar-se mais intensos com o odor característico destas refeições.

Observou-se, também, que outras causas multifatoriais, como a forma de apresentação das dietas hospitalares e o local de realização das refeições, podem estar envolvidas na menor aceitação dos alimentos pelas crianças.

Como conseqüência dessa menor aceitação, a ingestão alimentar desses pacientes pode estar nutricionalmente inadequada e ocasionar um comprometimento de seu estado nutricional e da eficácia do tratamento oncológico da LLA.

Dentro da situação proposta no estudo, e levando em consideração as observações e dados coletados durante a pesquisa, propõem-se algumas estratégias de intervenção nutricional que possam propiciar a essas crianças maior aceitação alimentar. Isso implica estender a atenção nutricional para além dos cuidados básicos com o valor nutricional das dietas, considerando, principalmente, seu aspecto gastronômico e contextual. 
Na literatura pesquisada, não existem relatos de intervenções nutricionais referentes ao aspecto gastronômico em hospitais e, ainda, faltam melhores dados acerca do tema Nutrição e Câncer. Sugere-se, pois, que mais estudos multidisciplinares, nessa área, sejam realizados, com a finalidade de investigar, mais atentamente, as causas multifatoriais envolvidas na alimentação e na aceitação do alimento por pacientes oncológicos. Assim, será possível uma intervenção clínica e nutricional mais completa no atendimento a esses pacientes.

\section{Nutritional proposals for children with cute lymphocytic leukemia under chemotherapy at the hospital of apoio in Brasília}

\section{Abstract}

The purpose of this study is to propose nutritional intervention which can increase the LLA infant patients' acceptance of hospital food, improving their health status, prognosis and life quality. In this research, it was analyzed a sample of 20 children from 1 to 10 years old who were under chemotherapy treatment in the Pediatrics' Division of Hematology at the Hospital of Support in Brasília (HAB). To the adults responsible for the children, it was applied a questionnaire about the food preference and eating habits of the LLA infant patients during the time they were hospitalized. The results of this study showed that during the time of hospitalization, snacks, such as fruit, yogurt and bread, were better accepted than meat, vegetables, rice and beans served during lunch and dinner. These findings suggest that the LLA infant patients' food intake is very poor and also insufficient to attend their energetic and nutritional needs, both factors which can compromise their health status and prognosis even more.

Key words: Leukemia. Nutritional therapy. Children. Nutrition.

\section{Referências}

ACCIOLY, E. et al. Nutrição em obstetrícia e pediatria. Rio de Janeiro: Cultura Médica, 2004.

BARACAT, F. et al. Cancerologia atual. São Paulo: Roca, 2000. 
BRASIL, A. L. D. et al. Nutrição e dietética em clínica pediátrica. São Paulo: Atheneu, 2003.

CUPPARI, L. Nutrição clínica no adulto. São Paulo: Manole, 2005.

IKEMORI, E. et al. Nutrição oncológica. São Paulo: Marina, 2003.

MAHAN, K. et al. Krause: alimentos, nutrição e dietoterapia. São Paulo: Roca, 2005.

MC GRATH, P. Reflections on nutritional issues associated with cancer therapy, Cancer Practice, United States, v. 10, n. 2, p. 94-101, 2002.

PINHO, N. B. et al. Manual de nutrição oncológica. Rio de Janeiro: Atheneu, 2004.

ROBBINS, Stanley et al. Patologia estrutural e funcional. Rio de Janeiro: Guanabara Koogan , 2000.

SHILS, Maurice et al. Tratado de nutrição moderna na saúde e na doença. São Paulo: Manole, 2002. v.2

STUMP, Sylvia et al. Nutrição relacionada ao diagnóstico e ao tratamento. 4. ed. São Paulo: Manole, 1999.

WAITZBERG, Dan. Dieta, nutrição e câncer. São Paulo: Atheneu, 2004. 
44 Univ. Ci. Saúde, Brasília, v. 5, n. 1/2, p. 35-49, 2007

Tatiana Serpa Guedes, Jaime Rodrigues Júnior e Bruna Toscano

APÊNDICE A - Modelo de questionário aplicado às crianças.

Idade (do filho):

Questionário

Sexo:

1. Das frutas abaixo, de quais o seu filho gosta?
( ) Banana
( ) Pêra
( ) Maçã
( ) Abacaxi
( ) Melancia
( ) Mamão
( ) Outras

2. Dos sucos abaixo, de quais o seu filho gosta?
( ) Laranja
( ) Caju
( ) Maracujá
( ) Limão
( ) Uva
( ) Pêssego
( ) Outros

3. Das bebidas abaixo, de quais o seu filho gosta?
( ) Refrigerante
( ) Água
( ) Café
( ) Suco de frutas ( ) Chá
( ) Vitamina de frutas
( ) Outras

4. Dos pães e biscoitos abaixo, de quais o seu filho gosta?

( ) Pão francês

( ) Pão de forma

Biscoito de sal

( ) Biscoito de maisena

( ) Bolacha de água e sal

Biscoito recheado

( ) Outros 
5. Dos cereais abaixo, de quais o seu filho mais gosta?

( ) Cereal matinal

( ) Pamonha

( ) Outros
( ) Arroz

( ) Macarrão
( ) Polenta

( ) Bolo comum

6. Dos vegetais abaixo, de quais o seu filho gosta?
( ) Brócolis
( ) Espinafre
( ) Couve
( ) Couve-flor
( ) Repolho
( ) Cenoura
( ) Beterraba
( ) Abóbora
( ) Vagem
( ) Chuchu
( ) Tomate
( ) Outros

7. Dos tipos de batata e mandioca abaixo, de quais o seu filho gosta?
( ) Batata cozida
( ) Batata frita
( ) Batata assada
( ) Purê de batata
( ) Mandioca frita
( ) Mandioca cozida
( ) Outros

8. Dos doces abaixo, de quais o seu filho gosta?
( ) Sorvete
( ) Pudim
( ) Gelatina
( ) Brigadeiro
( ) Bolo com cobertura ( ) Picolé
( ) Outros

9. Dos leites e derivados abaixo, de quais o seu filho gosta?
( ) Iogurte
（） Requeijão
( ) Queijo branco (minas, ricota)
( ) Queijo amarelo (mussarela, prato)
( ) Creme de leite
( ) Leite
( ) Outros 
10. Dos salgados abaixo, de quais o seu filho gosta?
( ) Pizza
( ) Coxinha de frango
( ) Misto-quente
( ) Hambúrguer
( ) Pipoca
( ) Cachorro-quente
( ) Outros

11. Das gorduras abaixo, de quais o seu filho gosta?
( ) Manteiga
( ) Margarina
( ) Maionese
( ) Azeite
( ) Óleo vegetal
( ) Outras

12. Das carnes abaixo, de quais o seu filho gosta?
( ) Peixe
( ) Frango
( ) Carne
( ) Lingüiça
( ) Salsicha
( ) Peru
( ) Outras

13. Dos tipos de carne abaixo, de quais o seu filho gosta?
( ) Estrogonofe
( ) Bife de panela
( ) Carne moída
( ) Almôndegas
( ) Picadinho
( ) Cozido de carne
( ) Outros

14. Dos tipos de ovos abaixo, de quais o seu filho gosta?
( ) Ovo cozido
( ) Ovo frito
( ) Ovo mexido
( ) Omelete
( ) Ovo de codorna
( ) Outros 
15. Das leguminosas abaixo, de quais o seu filho gosta?

$\begin{array}{lll}(\quad) \text { Feijão marrom } & (\quad \text { ) Feijão preto } & (\quad) \text { Ervilha } \\ (\quad) \text { Lentilha } & (\quad \text { ) Grão de bico } & (\quad \text { Soja } \\ (\quad) \text { Outras } & \end{array}$

16. O seu filho ainda mama?

( ) SIM ( ) NÃO

Se sim, com que freqüência?

( ) 1 vez ao dia

( ) 2 vezes ao dia

( ) 3 vezes ao dia

( ) Mais de 3 vezes ao dia

\section{APÊNDICE B - Modelo de questionário aplicado aos nutricionistas Questionário}

Nome do Hospital:

Quanto ao tratamento quimioterápico das crianças com LLA:

1. Quais são os efeitos colaterais de impacto nutricional mais comuns no tratamento de LLA?

2. O que é feito nutricionalmente para minimizá-los?

3. Em que grau esses efeitos interferem na alimentação da criança?

4. Em casos de mielosupressão, quando o paciente se apresenta em situação de maior risco de infecções, que conduta dietoterápica é adotada? 


\section{ANEXO A - Cardápio do hab}

\section{Cardápio do dia 01/01/07}

\begin{tabular}{|l|l|}
\hline REFEIÇÕES & ALIMENTOS \\
\hline Desjejum & $\begin{array}{l}\text { Café com leite } \\
\text { Pão francês com manteiga }\end{array}$ \\
\hline Almoço & Banana prata \\
\hline Tomate, beterraba e alface \\
Arroz \\
Feijão \\
Isca de frango \\
Vagem com milho sautée \\
Manga (sobremesa) \\
\hline Suco
\end{tabular}

\section{Cardápio do dia 02/01/07}

\begin{tabular}{|l|l|}
\hline REFEIÇÕES & ALIMENTOS \\
\hline Desjejum & $\begin{array}{l}\text { Leite achocolatado } \\
\text { Pão francês com manteiga }\end{array}$ \\
\hline Colação & Abacaxi \\
\hline Almoço & $\begin{array}{l}\text { Agrião, tomate e cenoura } \\
\text { Arroz } \\
\text { Feijão } \\
\text { Silveirinha de carne } \\
\text { Purê de batata }\end{array}$ \\
& $\begin{array}{l}\text { Melancia (sobremesa) } \\
\text { Suco }\end{array}$ \\
\hline
\end{tabular}


Univ. Ci. Saúde, Brasília, v. 5, n. 1/2, p. 35-49, 2007

Propostas dietoterápicas para crianças com leucemia linfocítica aguda ...

\begin{tabular}{|l|l|}
\hline Merenda & Leite achocolatado e bolo de laranja \\
\hline \multirow{3}{*}{ Jantar } & Sopa de caldo verde com frango \\
& $\begin{array}{l}\text { Arroz } \\
\text { Feijão } \\
\text { Frango em cubos grelhados } \\
\text { Abobrinha refogada }\end{array}$ \\
& $\begin{array}{l}\text { Salada de frutas (sobremesa) } \\
\text { Suco }\end{array}$ \\
\hline Ceia & Suco de Laranja e pão doce com cobertura \\
\hline
\end{tabular}

\section{Cardápio do dia 03/01/07}

\begin{tabular}{|c|c|}
\hline REFEIÇÕES & ALIMENTOS \\
\hline Desjejum & $\begin{array}{l}\text { Café com leite } \\
\text { Pão Francês com manteiga }\end{array}$ \\
\hline Colação & Mamão \\
\hline Almoço & $\begin{array}{l}\text { Alface, tomate, rabanete } \\
\text { Arroz } \\
\text { Feijão } \\
\text { Carne assada } \\
\text { Quibebe de abóbora } \\
\text { Melão (sobremesa) } \\
\text { Suco de tamarindo }\end{array}$ \\
\hline Merenda & $\begin{array}{l}\text { Suco de laranja com acerola e pão de } \\
\text { queijo }\end{array}$ \\
\hline Jantar & $\begin{array}{l}\text { Canja } \\
\text { Arroz } \\
\text { Feijão } \\
\text { Frango assado } \\
\text { Creme de milho } \\
\text { Melão (sobremesa) } \\
\text { Suco } \\
\end{array}$ \\
\hline Ceia & $\begin{array}{l}\text { Café com leite e pão francês com } \\
\text { manteiga }\end{array}$ \\
\hline
\end{tabular}


